$\mathrm{BNL}-79827-2008-\mathrm{CP}$

\title{
Vibration Measurements to Study the Effect of Cryogen Flow In a Superconducting Quadrupole
}

\author{
P. He, M. Anerella, S. Aydin, G. Ganetis, M. Harrison, A. Jain, \\ B. Parker
}

Presented at the Particle Accelerator Conference 2007 (PAC07)

June 25-29, 2007, Albuquerque, NM

January, 17, 2008

\author{
Superconducting Magnet Division \\ Brookhaven National Laboratory \\ P.O. Box 5000 \\ Upton, NY 11973-5000 \\ www.bnl.gov
}

Notice: This manuscript has been authored by employees of Brookhaven Science Associates, LLC under Contract No. DE-AC02-98CH10886 with the U.S. Department of Energy. The publisher by accepting the manuscript for publication acknowledges that the United States Government retains a non-exclusive, paid-up, irrevocable, world-wide license to publish or reproduce the published form of this manuscript, or allow others to do so, for United States Government purposes.

This preprint is intended for publication in a journal or proceedings. Since changes may be made before publication, it may not be cited or reproduced without the author's permission. 


\section{DISCLAIMER}

This report was prepared as an account of work sponsored by an agency of the United States Government. Neither the United States Government nor any agency thereof, nor any of their employees, nor any of their contractors, subcontractors, or their employees, makes any warranty, express or implied, or assumes any legal liability or responsibility for the accuracy, completeness, or any third party's use or the results of such use of any information, apparatus, product, or process disclosed, or represents that its use would not infringe privately owned rights. Reference herein to any specific commercial product, process, or service by trade name, trademark, manufacturer, or otherwise, does not necessarily constitute or imply its endorsement, recommendation, or favoring by the United States Government or any agency thereof or its contractors or subcontractors. The views and opinions of authors expressed herein do not necessarily state or reflect those of the United States Government or any agency thereof. 


\title{
VIBRATION MEASUREMENTS TO STUDY THE EFFECT OF CRYOGEN FLOW IN A SUPERCONDUCTING QUADRUPOLE*
}

\author{
P. He ${ }^{\#}$, M. Anerella, S. Aydin, G. Ganetis, M. Harrison, A. Jain, B. Parker \\ Superconducting Magnet Division, BNL, Upton, NY 11973, USA
}

\section{Abstract}

The conceptual design of compact superconducting magnets for the International Linear Collider final focus is presently under development. A primary concern in using superconducting quadrupoles is the potential for inducing additional vibrations from cryogenic operation. We have employed a Laser Doppler Vibrometer system to measure the vibrations in a spare RHIC quadrupole magnet under cryogenic conditions. Some preliminary results of these studies were limited in resolution due to a rather large motion of the laser head as well as the magnet. As a first step towards improving the measurement quality, a new set up was used that reduces the motion of the laser holder. The improved setup is described, and vibration spectra measured at cryogenic temperatures, both with and without helium flow, are presented.

\section{INTRODUCTION}

Compact superconducting quadrupoles have been proposed as promising candidates for the final focus region of the International Linear Collider (ILC) [1]. ILC will operate with nanometer scale beam size at the interaction point. This requires nanometer scale stability of the beam, and a similar mechanical stability in the final focus quadrupoles. One of the concerns in using superconducting magnets is the influence of the cryogen flow on the vibration characteristics of the magnets. As part of an ongoing R\&D effort at BNL to study the vibration behavior of superconducting quadrupoles, a spare RHIC arc quadrupole [2] was cooled down to $4.5 \mathrm{~K}$ and vibration measurements were made using a Laser Doppler Vibrometer system [3]. Some preliminary results showed that apart from a rather large ambient motion of the magnet, the measurement resolution was limited due to a rather large motion of the laser head itself [4]. To improve the measurement quality, we modified our setup to reduce the motion of the laser head. The new experimental setup and the vibration spectra obtained are described in this paper.

The RHIC arc quadrupoles are assembled along with a corrector package and either a sextupole or a trim quadrupole in a single cold mass assembly, referred to as a CQS assembly [2]. The cross section of the magnet cold

\footnotetext{
* This work was supported by the U.S. Department of Energy under contract number DE-AC02-98CH10886. This manuscript has been authored by Brookhaven Science Associates, LLC under Contract No. DE-AC02-98CH1 -886 with the U.S. Department of Energy. The United States Government retains, and the publisher, by accepting the article for publication, acknowledges, a worldwide license to publish or reproduce the published form of this manuscript, or allow others to do so, for the United States Government purposes.

\#phe@bnl.gov
}

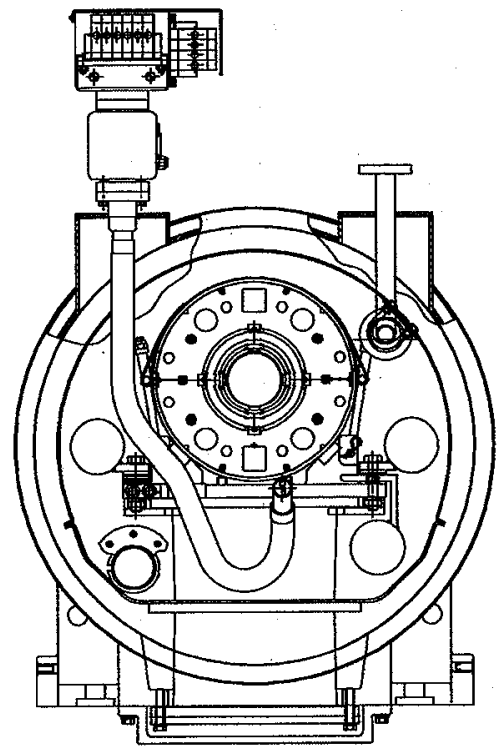

Figure 1: Cross section of a RHIC quadrupole cold mass in its cryostat.

mass in its cryostat is shown in Fig. 1. The cold mass is supported by vertical posts, but it is not constrained in the horizontal direction.

Given the constraints of cryogenic operation, and limited space available, it was not practical to use typical geophones or accelerometers. Instead, a non-contact optical interferometer based technique [3] was adopted. The performance of the laser system was tested against geophones in a series of special measurements [4]. These results showed that while good differential measurements can be made using the dual beam system, it is desirable to reduce the absolute motion of the laser holder as well as the magnet for obtaining better resolution.

\section{IMPROVED EXPERIMENTAL SETUP}

The laser holder used in our earlier studies of horizontal motion was attached to the CQS magnet test stand [4]. The motion of the magnet cryostat was quite large $(\sim 500 \mathrm{~nm}$ integrated above $1 \mathrm{~Hz})$ even though it was mounted on pneumatic isolation pads. These pads had a resonance frequency of $\sim 3-4 \mathrm{~Hz}$ and helped to minimize transmission of high frequency vibrations from the test stand to the magnet. However, when the magnet was hooked up to the cryogenic system, the connecting bellows were stiff enough to couple test stand motion to the magnet and then the isolation mounts were not very effective. To reduce the motion of the laser head, we detached the laser holder from the test stand and placed it on a heavy steel table, separated by $\sim 1.8 \mathrm{~m}$ from the CQS 


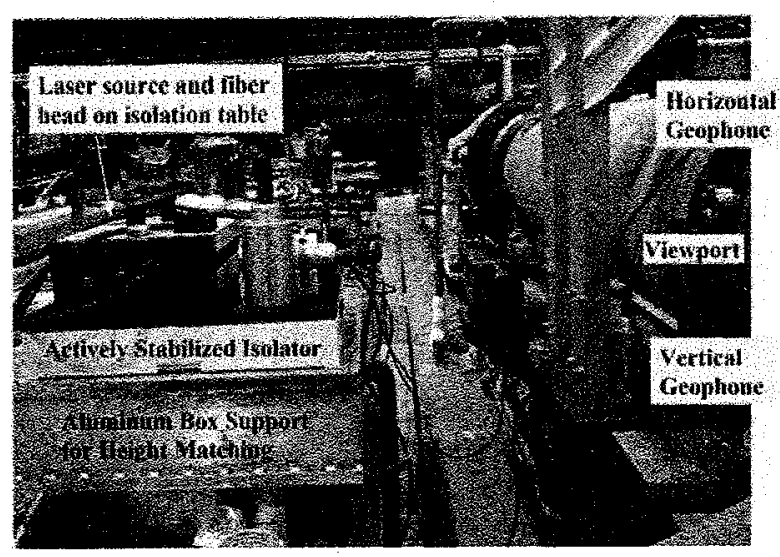

Figure 2: New laser setup for the CQS cold test.

test stand (see Fig. 2). This resulted in about an order of magnitude reduction in motion above $\sim 10 \mathrm{~Hz}$ (see Fig. 3). The absolute value of this motion, however, was still $-20 \mathrm{~nm}$. In order to further, reduce this motion, we installed the laser holder on top of an actively stabilized isolation table [5] with a resonance frequency less than $1 \mathrm{~Hz}$. This provided significant improvement, with the RMS motion now below $1 \mathrm{~nm}$ at $\geq 10 \mathrm{~Hz}$, as shown in Fig. 3.

New holders for the laser heads were built and installed on the isolation table, with the height matched to a view port on the cryostat through which the laser beam was directed on a retroreflecting target attached to the cold mass. Placing the laser interferometer unit also on the stabilized platform further reduced the signal noise. Two horizontal and two vertical geophones were used to monitor the motion of the laser holder and the magnet cryostat. A picture of the entire setup is shown in Fig. 2.

\section{MEASUREMENTS AND ANALYSIS}

The CQS magnet was hooked up to the cryogenic system and cooled down to $4.5 \mathrm{~K}$. Measurements of the horizontal motion of the cold mass were first made with helium flowing normally. The flow was then stopped from entering the magnet, with the magnet either isolated completely, or vented to the helium return line in order to avoid any pressure build up in the magnet. The entire sequence of Flow/No Flow (isolated or vented) measurements was repeated three times on the same day. Each measurement consisted of vibration data recorded at $1.6 \mathrm{kHz}$ for $143.5 \mathrm{~s} \mathrm{(} 224 \mathrm{~K}$ points). Fourier analysis of one data set was performed in blocks of $16 \mathrm{~K}$ points, giving a frequency resolution of $\sim 0.1 \mathrm{~Hz}$. The power spectra from all the blocks of $16 \mathrm{~K}$ points in a given data set were averaged to reduce uncertainties in the spectra. The measurements were repeated almost continuously in order to monitor any changes in the motion with time. The helium pressure in the magnet was also recorded along with the vibration data. All the power spectra were then averaged over many runs throughout the day for better statistics. All measurements were made in the single beam mode of the dual beam Laser Doppler Vibrometer, which essentially gives the absolute motion of the cold mass,

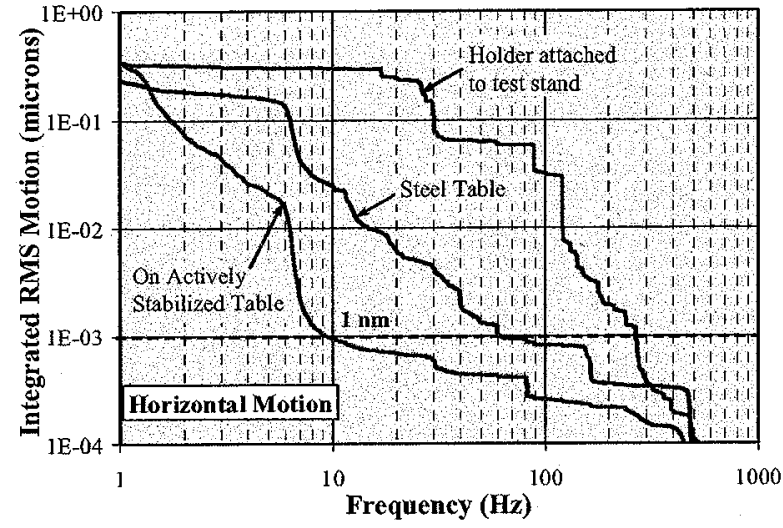

Figure 3: Laser holder motion in the earlier setup, and in the new setup with and without stabilization. The curves give the total RMS motion at or above any given frequency. Steps in the curves correspond to prominent peaks in the power spectrum.

assuming that the motion of the laser head can be neglected in comparison to the magnet motion.

The integrated spectra obtained with and without helium flow are shown in Fig. 4. In order to get an overall picture, these spectra are averaged over all runs of a given type during the day. A typical spectrum for the laser head motion is also shown for comparison. At very low frequencies (below $\sim 8 \mathrm{~Hz}$ ), the motion of the laser head itself becomes significant. Nevertheless, it remains at least a factor of two below the motion of the magnet.

The RMS motion above $1 \mathrm{~Hz}$ is about the same with or without the flow with the magnet isolated, although the power spectra differ slightly in detail. Particularly noteworthy is the case when the magnet is vented, giving rise to two very prominent peaks at $\sim 8 \mathrm{~Hz}$ and $\sim 10.5 \mathrm{~Hz}$. Also, the peak at $\sim 5.5 \mathrm{~Hz}$ is much more prominent when the magnet is vented. The net result is that the RMS motion is much larger in the vented case.

To study the behavior of various peaks with time, the RMS motion in a band between two frequencies, $f_{1}$ and $f_{2}$, was obtained by integrating the power spectra from $f_{1}$ to $f_{2}$. Fig. 5 shows the total motion above $1 \mathrm{~Hz}$ from each of

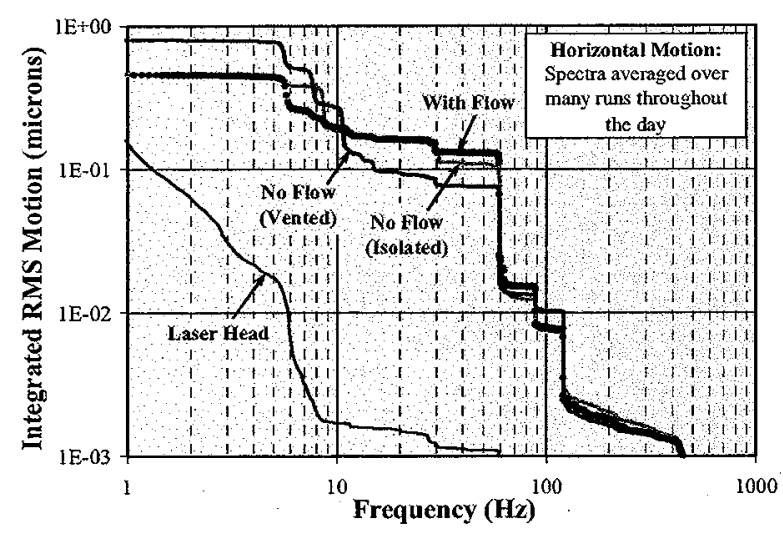

Figure 4: Integrated spectra with and without helium flow, averaged over all runs during the day. 


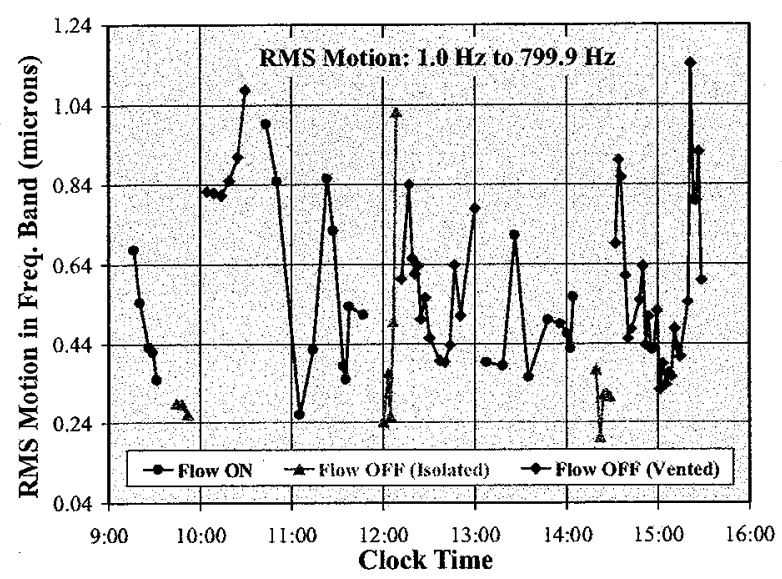

Figure 5: Cold mass RMS motion above $1 \mathrm{~Hz}$.

the measurements during the day. It can be seen that the motion varies a lot from run to run, but it is generally slightly less with the flow off and with the magnet isolated, except for an odd point at 12:08 PM. The motion always increases when the magnet is vented, primarily due to a prominent peak at $\sim 10.5 \mathrm{~Hz}$. The behavior of this peak can be studied by looking at RMS motion in a narrow band of $10 \mathrm{~Hz}$ to $13.5 \mathrm{~Hz}$. As can be seen from Fig. 6, large motion is induced in this band when the magnet is vented. However, this motion decays with time and practically disappears after a period of about half an hour. The exact mechanism that excites this motion is not clear.

The helium pressure in the magnet was also recorded along with the vibration data to look for any correlation between magnet motion and pressure fluctuations. Typical power spectra for pressure fluctuations are given in Fig. 7 . When the helium is flowing through the magnet, there is a prominent $(\sim 10 \mathrm{psi}$, or $69 \mathrm{kPa}$ peak-to-peak) pressure fluctuation at $8.1 \mathrm{~Hz}$. Several higher order harmonics can be seen as well. When the flow is stopped, but the magnet is not vented, the pressure fluctuations still persist, although the frequencies are slightly shifted and the peakto-peak variation is slightly less. The pressure is practically constant when the magnet is vented to the

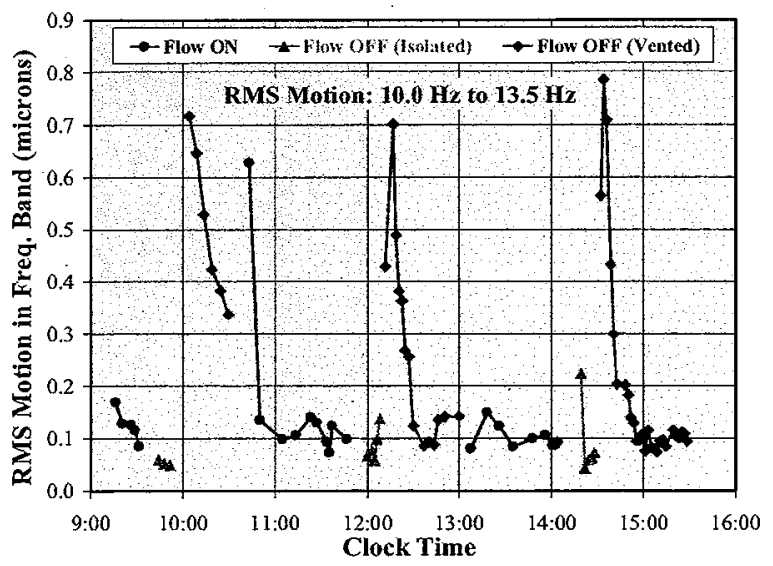

Figure 6: Motion in the frequency band of $10.0 \mathrm{~Hz}$ to $13.5 \mathrm{~Hz}$.

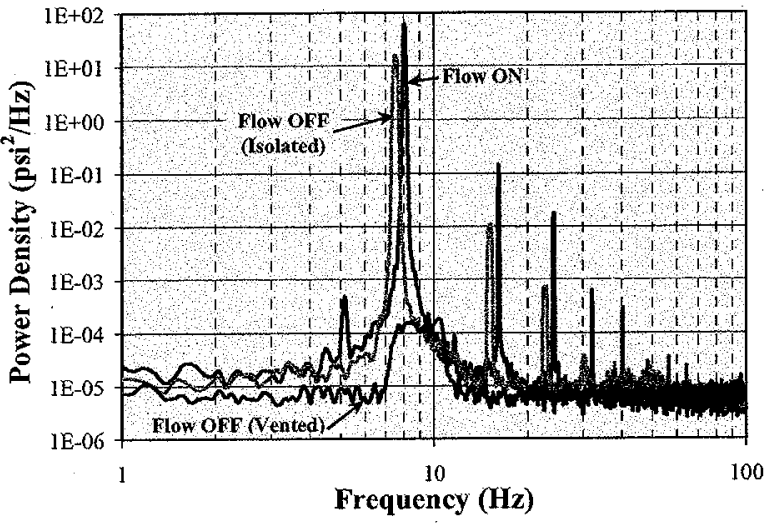

Figure 7: Power density spectra of helium pressure variations in the magnet with and without flow.

helium return line. No direct correlation between helium pressure fluctuations and magnet motion was found.

\section{SUMMARY}

The vibrations in a superconducting RHIC quadrupole have been measured with and without helium flow in order to investigate the effect of cryogen flow on the magnet stability. With an improved experimental setup, including the use of an actively stabilized isolation table to mount the laser heads, the measurement errors have been reduced to less than a few $\mathrm{nm}$ above $\sim 8 \mathrm{~Hz}$. This allowed us to measure the absolute motion of the cold mass (single beam mode) instead of motion relative to the cryostat, which itself is not steady. When the helium flow is stopped, but the magnet is not vented, the motion reduces very slightly in nearly all frequency bands. However, the magnet pressure builds up in this mode as the magnet warms. When the magnet is vented, a strong peak (several hundred nm RMS) shows up at $\sim 10-11 \mathrm{~Hz}$, but this motion decays with time. These results appear quite encouraging for the use of superconducting technology for the quadrupoles in the final focus region of the ILC. The techniques developed in this study will also be useful to measure the vibration behavior of a realistic ILC final focus quadrupole when a prototype becomes available.

\section{REFERENCES}

[1] B. Parker, et al., "The Superconducting Magnets of the ILC Beam Delivery System," these proceedings, paper THPMS091.

[2] M. Anerella, et al., "The RHIC Magnet System,", Nucl. Instrum. Meth. A499(2-3), pp. 280-315(2003)

[3] PolyTec Inc. http://www.polytec.com, model OFV552 fiber-optic head and model OFV-5000 controller.

[4] A. Jain, et al., "Vibration Measurements in a RHIC Quadrupole at Cryogenic Temperatures," Nanobeam2005, Kyoto, Japan, Oct.17-21, 2005; paper WG2d-05.

[5] Kinetic System Inc. http://www.kineticsystems.com, model KSI 8001. 